УДК 616.381-009.7+615.317]-085-053.2

DOI: 10.26435/UC.V0I3(36).510

\author{
А.В. Налетов, Д.А Карпенко
}

ГОО ВПО «Донецкий национальный медицинский университет имени М. Горького», Донецк

\title{
ЭФФЕКТИВНОСТЬ ИСПОЛЬЗОВАНИЯ В. INFANTIS 35624 В ЛЕЧЕНИИ СИНДРОМА РАЗДРАЖЕННОГО КИШЕЧНИКА
}

Функциональные гастроинтестинальные расстройства (ФГИР) занимают одно из ведущих мест в структуре патологии органов пищеварения среди пациентов разных возрастных групп. Длительное рецидивирующее течение ФГИР, неэффективное лечение приводят к значительному снижению качества жизни, длительной ограниченности в приеме ряда продуктов питания и напитков, изменению туалетных привычек, что обязательно сказывается на психологическом состоянии больного, усугубляя течение заболевания, разочаровывая пациента в отношении к лечению, снижая его комплаентность, а в глобальном смысле приводит к существенному экономическому ущербу для системы здравоохранения за счет прямых и косвенных расходов на лечение таких больных $[1,2]$. Одним из наиболее распространенных в популяции ФГИР является синдром раздраженного кишечника (СРК). Частота встречаемости СРК у детей разных стран находится в диапазоне 1,2-5,4\% [3], а среди взрослых пациентов этот показатель составляет примерно 11,2\% [4, 5].

СРК с современных позиций рассматривается как мультифакториальное расстройство. Результирующие патофизиологические механизмы включают нарушения моторики, висцеральную гиперчувствительность, повышенную кишечную проницаемость, иммунную активацию, дисбаланс кишечной микробиоты и расстройство функционирования оси «мозг-кишечник» [4-6].

За последние годы возрос интерес к возможной роли микробиоты кишечника в патогенезе ФГИР [6]. Способность кишечной микробиоты вырабатывать нейротрансмиттеры, изменяющие при этом секрецию и моторику кишечника, а также порог висцеральной чувствительности и барьерные свойства слизистой оболочки свидетельствует о существенной роли дисбиотических изменений в патогенезе СРК [7]. В аналитических обзорах и метаанализах, посвященных роли микробиоты кишечника в формировании СРК, а также в материалах «Римского кон- сенсуса IV» отмечается, что изменение состава кишечной микробиоты может рассматриваться в качестве одного из звеньев патогенеза СРК, изменяя нейромоторные сенсорные, барьерные функции кишечника и их взаимодействие по оси «головной мозг - кишечник» [3-5].

Использование пробиотиков является перспективным методом лечения пациентов с ФГИР. Пробиотики - живые микроорганизмы, которые при применении в адекватных количествах вызывают улучшение здоровья организма хозяина. Пробиотические бактерии обладают множеством положительных свойств. Они непатогенны для человека, выдерживают прохождение через кислую среду желудка, могут адгезироваться к эпителиальным клеткам слизистой оболочки кишечника и колонизировать их. Пробиотики также модулируют иммунитет желудочно-кишечного тракта путем изменения уровня цитокинов. Существует множество видов пробиотиков, но не все из них доказали свою эффективность. На сегодняшний день все более актуальной становится стратегия штаммоспецифичности использования пробиотиков не только при лечении заболеваний пищеварительного тракта, но и при патологии других органов и систем.

В настоящее время Bifidobacterium infantis 35624 имеет лучшую доказательную базу эффективности применения при СРК и рекомендован «Римским консенсусом IV» для лечения пациентов с данной патологией [3-5]. Доказано, что В. infantis 35624, широко изучаемый два десятилетия, оказывает целенаправленное действие на толстую кишку. Согласно рекомендациям Всемирной организации здравоохранения, продемонстрированные преимущества использования пробиотиков применимы только к данному специфическому штамму, но не к общему роду или виду и не к пробиотикам в целом [8-10].

(c) А.В. Налетов, Д.А Карпенко, 2020

(c) Университетская Клиника, 2020 
B. infantis 35624 - натуральный бактериальный штамм, который изначально был выделен из терминального отдела подвздошной кишки здоровой 70-летней женщины. Поскольку эта женщина была субъектом под номером 356 в исследовании, проведенном компанией Alimentary Health, а выбранный изолят был 24-м, штамм получил номер 35624. В 2016 году при секвенировании и сравнении полного генома данного микроорганизма с другими геномами бактерий рода Bifidobacterium была обнаружена идентичность штаммов В. infantis 35624 и В. longum 35624.

В исследовании при изучении фекального выделения B. infantis 35624 с помощью метода полимеразной цепной реакции до и после 8 недель ежедневного приема пробиотика, продемонстрирована способность микроорганизма преодолевать в капсулированной форме агрессивные среды желудка и тонкой кишки [11].

K важным свойствам B. infantis 35624 можно отнести способность пробиотика индуцировать иммуномодулирующие эффекты, что показано в ряде исследований на животных и у пациентов с патологией кишечника.

На первоначальном этапе исследований оценки эффективности В. infantis 35624 в эксперименте на крысах было обнаружено несколько противовоспалительных свойств данной бактерии. Так, крысы с воспалением толстой кишки на фоне отсутствия интерлейкина (ИЛ) 10 , получали В. infantis 35624 или плацебо в течение 19 недель. По завершении исследования у крыс, получавших пробиотик, воспалительный процесс в желудочно-кишечном тракте был значительно менее выражен, чем в группе, получавших плацебо [12].

При культивации эпителиальных клеток, полученных из толстой кишки человека, с B. infantis 35624 было отмечено уменьшение уровня ИЛ-8 по сравнению с исходными значениями [13].

В исследованиях, проведенных O’Mahony и соавторами, продемонстрировано, что прием B. infantis 35624 может изменить уровни цитокинов у больных с СРК. Так, установлено, что B. infantis 35624 индуцировал продукцию противовоспалительных цитокинов - ИЛ-10, мононуклеарами мезентериальных лимфатических узлов и периферической крови у пациентов с активным колитом [14]. В другом исследовании у здоровых добровольцев также было показано, что прием B. infantis 35624 в течение 8 недель ассоциирован со значительным увеличением уровня ИЛ-10 [15].

Иммуномодулирующее и противовоспалительное свойства B. infantis 35624 являются ак- туальными для пациентов с СРК, поскольку современные данные подтверждают наличие минимального воспаления слизистой оболочки кишечника при СРК, которое может служить причиной развития висцеральной гиперчувствительности. Кроме этого, эти свойства позволяют предполагать эффективность B. infantis 35624 и при лечении воспалительных заболеваний кишечника (болезнь Крона, язвенный колит).

Ряд исследований продемонстрировал эффективность и безопасность применения В. infantis 35624 в коррекции основных симптомов СРК у взрослых пациентов [16-19].

В работе P.J. Whorwell и соавторов было проведено двойное слепое, плацебо-контролируемое, многоцентровое клиническое исследование, оценивающее эффективность В. infantis 35624 в лечении СРК и подборе оптимальной дозировки пробиотика. Было обследовано 362 женщины с симптомами СРК. Установлено значительные улучшения на 4-й неделе лечения в группе пациентов, получавших B. infantis 35624 в дозе $108 \mathrm{KOE} /$ мл, по таким показателям, как вздутие живота, ощущение неполного опорожнения кишечника, натуживание, удовлетворение дефекацией [18]. Также в данном исследовании отмечено улучшение общего самочувствия и качества жизни пациентов с использованием опросника IBS-QOL. Данные показатели в группе пациентов, получавших В. infantis 35624, были на $20 \%$ выше. Полученные результаты показывает, что улучшение негастроэнтерологических проявлений СРК, которые рутинно не оцениваются в клинических исследованиях и в практической работе врача, на фоне терапии B. infantis 35624 являются важным составляющим для повышения удовлетворенности пациентов лечением.

В двух исследованиях, проведенных на крысах, показано, что использование пробиотика B. infantis 35624 способствует уменьшению висцеральной гиперчувствительности и, соответственно, висцеральной боли при воспалении кишечника [20,21].

Противовоспалительный эффект B. infantis 35624 подтвержден в исследовании D. Groeger, где отмечено, что пероральный прием пробиотика в течение 6-8 недель снижает повышенный уровень маркеров воспаления (С-реактивный белок, фактор некроза опухоли альфа и ИЛ-6) у пациентов с язвенным колитом. В отличие от пациентов с воспалительными заболеваниями кишечника, получающих В. infantis 35624, у здоровых добровольцев, принимавших В. infantis 35624, и пациентов с язвенным колитом, получающих плацебо, уровни изучаемых воспалительных маркеров в плазме крови не изменились [22]. 
Возможным объяснением противовоспалительного и иммуномодулирующего эффектов бактерии является наличие на поверхности В. infantis 35624 специфического экзополисахарида (EPS624), способного модулировать секрецию цитокинов и снижать активацию транскрипционного фактора NF-кB [23, 24].

В многоцентровом, двойном слепом, рандомизированном, плацебо-контролируемом исследовании, проведенном T. Ringel-Kulka и соавторами в 2016 году, оценивались эффекты В. infantis 35624 у добровольцев с жалобами на дискомфорт и метеоризм. В исследование было включено 302 человека, которые отмечали дискомфорт в области живота и вздутие чаще, чем 2 раза в неделю, по крайней мере в течение 3 месяцев. При этом участники исследования ранее не обращались к врачу и не получали никакого лечения по этому поводу в течение последние 12 месяцев. Испытуемые получали пробиотик В. infantis 35624 в дозе $109 \mathrm{KOE} /$ мл в виде капсул $(\mathrm{n}=143)$ или плацебо $(\mathrm{n}=154)$ длительностью 4 недели. Обе группы показали статистически значимые отличия ( $\mathrm{p}<0,001)$ по уменьшению дискомфорта и вздутия живота после 4-недельного периода лечения. Не выявлено значимых различий по сумме баллов при оценке симптомов на момент завершения исследования между группой, получавшей пробиотик и плацебо. Однако, частота «бессимптомных» дней была на 72\% больше в группе, принимающей B. infantis 35624, по сравнению с группой, получающей плацебо (p<0,035) [25].

В другом рандомизированном двойном слепом плацебо-контролируемом исследовании, проведенном O’Mahony, принимали участие 77 мужчин и женщин с СРК с различными подтипами. В исследование проведено сравнение влияния приема в течение 8 недель L. salivarius UCC4331 и B. infantis 35624 на симптомы заболевания и уровни цитокинов. В ходе работы были оценены симптомы СРК (боль в животе, вздутие, нарушение перистальтики), качество жизни, оценена частота и консистенция стула, проведен микробиотический анализ, изучены уровни ИЛ-10 и ИЛ-12 в сыворотке крови. Прием B. infantis 35624 в течение 8 недель обеспе- чил достоверное уменьшение функциональной боли в животе, неприятных ощущений и вздутия живота, проблем со стулом, а также уменьшение общей симптоматики. Эффективность В. infantis 35624 отмечена у всех пациентов независимо от подтипа СРК. При этом позитивная клиническая динамика сопровождалась повышением содержания в сыворотке крови противовоспалительного цитокина ИЛ-10 до уровня здоровых лиц и нормализацией соотношения ИЛ-10/ИЛ-12. Применяемый в качестве сравнения пробиотик L. salivarius UCC4331 не показал достоверного клинического и лабораторного улучшения [16].

В проведенных исследованиях отмечается хорошая переносимость B. infantis 35624 на протяжении периода всего наблюдения. Не отмечено каких-либо серьезных нежелательных эффектов от лечения.

В одном из последних метаанализов, проведенном американскими исследователями из США, отмечено, что пробиотики, содержащие в своем составе B. infantis 35624, являются эффективными в лечении СРК у взрослых. При этом, применение B. infantis 35624 позволяет существенно облегчить симптомы заболевания (боли либо неприятные ощущения в животе; вздутие/ метеоризм; расстройства стула - диарея/запор; чувство неполного опорожнения кишечника и неотхождения газов; натуживание и ряд других проявлений, в том числе и негастроэнтерологических) без развития побочных эффектов от лечения [26].

Таким образом, учитывая противовоспалительный и иммуномодулирующий эффект, использование B. infantis 35624 в лечении пациентов с СРК способствует эффективному купированию основных симптомов заболевания. Однако следует отметить, что работы по применению данного пробитика остаются немногочисленными, а в лечении пациентов детского возраста исследования по этому вопросу отсутствуют, что обусловливает актуальность дальнейшего изучения данного направления терапии пациентов с СРК и в том числе в педиатрической практике. 


\section{А.В. Налетов, Д.А Карпенко}

ГОО ВПО «Донецкий национальный медицинский университет имени М.Горького», Донецк

\section{ЭФФЕКТИВНОСТЬ ИСПОЛЬЗОВАНИЯ В. INFANTIS 35624 В ЛЕЧЕНИИ СИНДРОМА РАЗДРАЖЕННОГО КИШЕЧНИКА}

Одним из наиболее распространенных в популяции функциональных гастроинтестинальных расстройств является синдром раздраженного кишечника. За последние годы возрос интерес к возможной роли микробиоты кишечника в патогенезе функциональных гастроинтестинальных расстройств. Использование пробиотиков является перспективным методом лечения пациентов с данной патологией. В статье представлены современные литературные данные об эффективности применения пробиотика Bifidobacterium infantis 35624 в купировании основных клинических проявлений синдрома раздраженного кишечника, в результате противовоспалительного и иммуномодулирующего свойств пробиотика.

Ключевые слова: синдром раздраженного кишечника, пробиотик, Bifidobacterium infantis 3562.

\section{A.V. Nalyotov, D.A. Karpenko}

\section{SEI HPE «M. Gorky Donetsk National Medical University», Donetsk}

\section{EFFECTIVENESS OF B. INFANTIS 35624 IN THE TREATMENT OF IRRITABLE BOWEL SYNDROME}

Irritable bowel syndrome is one of the most common functional gastrointestinal disorders in the population. In recent years, the interest of the possible role of the gut microbiota in the pathogenesis of functional gastrointestinal disorders has increased. The use of probiotics is a promising method of treating patients with this pathology. The article presents modern literature data on the effectiveness of probiotic Bifidobacterium infantis 35624 in relieving the main clinical manifestations of irritable bowel syndrome, due to the anti-inflammatory and immunomodulatory properties of the probiotic.

Key words: irritable bowel syndrome, probiotic, Bifidobacterium infantis 35624 .

\section{ЛИТЕРАТУРА}

1. Печкуров Д.В. Возрастные особенности функциональных расстройств желудочно-кишечного тракта, проявляющихся абдоминальными болями, с позиций биопсихосоциальной модели. Вопросы детской диетологии. 2015; 13 (2): 11-16.

2. Налетов А.В., Вьюниченко Ю.С., Коктышев И.В. Оценка качества жизни детей с синдромом раздраженного кишечника, проживающих в условиях военного конфликта в Донбассе. Университетская клиника. 2018; 2(27): 12-16.

3. Hyams J. S., Di Lorenzo C., Saps M., et al. Functional disorders: children and adolescents. Gastroenterology. 2016; 150: $1456-1468 \mathrm{e} 2$.

4. Drossman D.A., Hasler W.L. Rome IV - Functional GI disorders: disorders of Gut-Brain interaction. Gastroenterology. 2016; 150(6): 1262-79.

5. Lacy B.E., Mearin F., Chang L., et al. Bowel Disorders. Gastroenterology. 2016; 150: 1393-1407.

6. Налетов А.В., Вьюниченко Ю.С., Лянник В.А. Особенности течения синдрома раздраженного кишечника у детей, проживающих в условиях военного конфликта в Донбассе. Университетская клиника. 2018; 1 (26): 5458 .

7. Трухан Д.И., Голошубина В.В., Иванова Д.С. Актуальные вопросы диагностики и лечения синдрома раздраженного кишечника. Медицинский совет. 2018; 21 : 110-116.

8. Gerritsen J., Smidt H., Rijkers G.T., de Vos W.M. Intestinal microbiota in human health and disease: the impact of probiotics. Genes Nutr. 2011; 6: 209-240.

9. Distrutti E., Monaldi L., Ricci P., Fiorucci S. et al. Gut microbiota role in irritable bowel syndrome: New therapeutic strategies. World J. Gastroenterol. 2016. 22 (7): 22192241.

\section{REFERENCES}

1. Pechkurov D.V. Vozrastnye osobennosti funkcional'nyh rasstrojstv zheludochno-kishechnogo trakta, projavljajushhihsja abdominal'nymi boljami, s pozicij biopsihosocial'noj modeli. Voprosy detskoj dietologii. 2015; 13 (2): 11-16. (in Russian).

2. Naletov A.V., V'junichenko Ju.S., Koktyshev I.V. Ocenka kachestva zhizni detej s sindromom razdrazhennogo kishechnika, prozhivajushhih v uslovijah voennogo konflikta v Donbasse. Universitetskaja klinika. 2018; 2(27): 1216. (in Russian).

3. Hyams J. S., Di Lorenzo C., Saps M., et al. Functional disorders: children and adolescents. Gastroenterology. 2016; 150: $1456-1468 \mathrm{e} 2$.

4. Drossman D.A., Hasler W.L. Rome IV - Functional GI disorders: disorders of Gut-Brain interaction. Gastroenterology. 2016; 150(6): 1262-79.

5. Lacy B.E., Mearin F., Chang L., et al. Bowel Disorders. Gastroenterology. 2016; 150: 1393-1407.

6. Naletov A.V., V'junichenko Ju.S., Ljannik V.A. Osobennosti techenija sindroma razdrazhennogo kishechnika $u$ detej, prozhivajushhih $\mathrm{v}$ uslovijah voennogo konflikta v Donbasse. Universitetskaja klinika. 2018; 1 (26): 54-58. (in Russian).

7. Truhan D.I., Goloshubina V.V., Ivanova D.S. Aktual'nye voprosy diagnostiki i lechenija sindroma razdrazhennogo kishechnika. Medicinskij sovet. 2018; 21: 110-116. (in Russian).

8. Gerritsen J., Smidt H., Rijkers G.T., de Vos W.M. Intestinal microbiota in human health and disease: the impact of probiotics. Genes Nutr. 2011; 6: 209-240.

9. Distrutti E., Monaldi L., Ricci P., Fiorucci S. et al. Gut microbiota role in irritable bowel syndrome: New therapeutic strategies. World J. Gastroenterol. 2016. 22 (7): 22192241. 
10. Bull M.J., Plummer N.T. Part2: Treatment for chronic gastrointestinal disease and gut dysbiosis. Integr. Med (Encinitas). 2015; 14 (1): 25-33.

11. Charbonneau D., Gibb R.D., Quigley E.M. Fecal excretion of Bifidobacterium infantis 35624 and changes in fecal microbiota after eight weeks of oral supplementation with encapsulated probiotic. Gut Microbes. 2013; 4 (3): 201211.

12. McCarthy J., O’Mahony L., O'Callaghan L., et al. Double blind, placebo controlled trial of two probiotic strains in interleukin 10 knockout mice and mechanistic link with cytokine balance. Gut. 2003; 52: 975-980.

13. Jobin C., Sartor R. B. The I kappa B/NF-kappa B system: a key determinant of mucosal inflammation and protection. Am. J. Physiol. Cell Physiol. 2000; 278: C451-C462.

14. O`Mahony L., O'Callaghan L., McCarthy J., et al. Differential cytokine response from dendritic cells to commensal and pathogenic bacteria in different lymphoid compartments in humans. Am. J. Physiol. Gastrointest. Liver Physiol. 2006; 290 (4): G 839-845.

15. Konieczna P., Groeger D., Ziegler M., et al. Bifidobacterium infantis 35624 administration induces Foxp3 T regulatory cells in human peripheral blood: potential role for myeloid and plasmacytoid dendritic cells. Gut. 2012; 61 (3): 354-366.

16. O'Mahony L., McCarthy J., Kelly P., et al. Lactobacillus and Bifidobacterium in irritable bowel syndrome: symptom responses and relationship to cytokine profiles. Gastroenterology. 2005; 128: 541-551.

17. McFarland L.V., Dublin S. Meta-analysis of probiotics for the treatment of irritable bowel syndrome. World J. Gastroenterol. 2008; 17: 2650-2661.

18. Whorwell P.J., Altringer L., Morel J., et al. Efficacy of an encapsulated probiotic Bifidobacterium infantis 35624 in women with irritable bowel syndrome. Am. J. Gastroenterol. 2006; 101 (7): 1581-1590.

19. Aragon G., Graham D.B., Borum M., Doman D.B. Probiotic therapy for irritable bowel syndrome. Gastroenterol. Hepatol. (NY). 2010; 6 (1): 39-44.

20. Johnson A.C., Greenwood-Van Meerveld B., McRorie J. Effects of Bifidobacterium infantis 35624 on post-inflammatory visceral hypersensitivity in the rat. Dig. Dis. Sci. 2011; 56 (11): 3179-3186.

21. McKernan D.P., Fitzgerald P., Dinan T.G., Cryan J.F. The probiotic Bifidobacterium infantis 35624 displays visceral antinociceptive effects in the rat. Neurogastroenterol. Motil. 2010; 22 (9): 1029-1035.

22. Groeger D., O'Mahony L., Murphy E.F., et al. Bifidobacterium infantis 35624 modulates host inflammatory processes beyond the gut. Gut Microbes. 2013; 4 (4): 325-339.

23. Altmann F., Kosma P., O'Callaghan A., et al. Genome analysis and characterisation of the exopolysaccharide produced by Bifidobacterium longum subsp. longum $35624^{\mathrm{TM}}$. PLoS One. 2016; 11(9): e0162983.

24. Schiavi E., Plattner S, Rodriguez-Perez N, et al. Exopolysaccharide from Bifidobacterium longum subsp. longum 35624 modulates murine allergic airway responses. Benef Microbes. 2018; 9 (5): 761-773.

25. Ringel-Kulka T., McRorie J, Ringel Y. Multi-center, double-blind, randomized, placebo-controlled, parallel-group study to evaluate the benefit of the probiotic Bifidobacterium infantis 35624 in non-patients with symptoms of abdominal discomfort and bloating. Am. J. Gastroenterol. 2017; 112 (1): 145-151.

26. Yuan F., Ni H., Asche C.V., et al. Efficacy of Bifidobacterium infantis 35624 in patients with irritable bowel syndrome: a meta-analysis. Curr. Med. Res. Opin. 2017; 33 (7): 1191-1197.
10. Bull M.J., Plummer N.T. Part2: Treatment for chronic gastrointestinal disease and gut dysbiosis. Integr. Med (Encinitas). 2015; 14 (1): 25-33.

11. Charbonneau D., Gibb R.D., Quigley E.M. Fecal excretion of Bifidobacterium infantis 35624 and changes in fecal microbiota after eight weeks of oral supplementation with encapsulated probiotic. Gut Microbes. 2013; 4 (3): 201211.

12. McCarthy J., O’Mahony L., O'Callaghan L., et al. Double blind, placebo controlled trial of two probiotic strains in interleukin 10 knockout mice and mechanistic link with cytokine balance. Gut. 2003; 52: 975-980.

13. Jobin C., Sartor R. B. The I kappa B/NF-kappa B system: a key determinant of mucosal inflammation and protection. Am. J. Physiol. Cell Physiol. 2000; 278: C451-C462.

14. O`Mahony L., O’Callaghan L., McCarthy J., et al. Differential cytokine response from dendritic cells to commensal and pathogenic bacteria in different lymphoid compartments in humans. Am. J. Physiol. Gastrointest. Liver Physiol. 2006; 290 (4): G 839-845.

15. Konieczna P., Groeger D., Ziegler M., et al. Bifidobacterium infantis 35624 administration induces Foxp3 T regulatory cells in human peripheral blood: potential role for myeloid and plasmacytoid dendritic cells. Gut. 2012; 61 (3): 354-366.

16. O’Mahony L., McCarthy J., Kelly P., et al. Lactobacillus and Bifidobacterium in irritable bowel syndrome: symptom responses and relationship to cytokine profiles. Gastroenterology. 2005; 128: 541-551.

17. McFarland L.V., Dublin S. Meta-analysis of probiotics for the treatment of irritable bowel syndrome. World J. Gastroenterol. 2008; 17: 2650-2661.

18. Whorwell P.J., Altringer L., Morel J., et al. Efficacy of an encapsulated probiotic Bifidobacterium infantis 35624 in women with irritable bowel syndrome. Am. J. Gastroenterol. 2006; 101 (7): 1581-1590.

19. Aragon G., Graham D.B., Borum M., Doman D.B. Probiotic therapy for irritable bowel syndrome. Gastroenterol. Hepatol. (NY). 2010; 6 (1): 39-44.

20. Johnson A.C., Greenwood-Van Meerveld B., McRorie J. Effects of Bifidobacterium infantis 35624 on post-inflammatory visceral hypersensitivity in the rat. Dig. Dis. Sci. 2011; 56 (11): 3179-3186.

21. McKernan D.P., Fitzgerald P., Dinan T.G., Cryan J.F. The probiotic Bifidobacterium infantis 35624 displays visceral antinociceptive effects in the rat. Neurogastroenterol. Motil. 2010; 22 (9): 1029-1035.

22. Groeger D., O’Mahony L., Murphy E.F., et al. Bifidobacterium infantis 35624 modulates host inflammatory processes beyond the gut. Gut Microbes. 2013; 4 (4): 325-339.

23. Altmann F., Kosma P., O’Callaghan A., et al. Genome analysis and characterisation of the exopolysaccharide produced by Bifidobacterium longum subsp. longum $35624^{\mathrm{TM}}$. PLoS One. 2016; 11(9): e0162983.

24. Schiavi E., Plattner S, Rodriguez-Perez N, et al. Exopolysaccharide from Bifidobacterium longum subsp. longum 35624 modulates murine allergic airway responses. Benef Microbes. 2018; 9 (5): 761-773.

25. Ringel-Kulka T., McRorie J, Ringel Y. Multi-center, double-blind, randomized, placebo-controlled, parallel-group study to evaluate the benefit of the probiotic Bifidobacterium infantis 35624 in non-patients with symptoms of abdominal discomfort and bloating. Am. J. Gastroenterol. 2017; 112 (1): 145-151.

26. Yuan F., Ni H., Asche C.V., et al. Efficacy of Bifidobacterium infantis 35624 in patients with irritable bowel syndrome: a meta-analysis. Curr. Med. Res. Opin. 2017; 33 (7): 1191-1197. 